\title{
Towards Organisational Learning Enhancement: Assessing Software Engineering Practice
}

\author{
Sufian Fannoun, John Kerins \\ Department of Computer Science \\ Faculty of Science and Engineering \\ University of Chester \\ Chester, United Kingdom \\ e-mail: s.fannoun@chester.ac.uk,j.kerins@chester.ac.uk
}

\begin{abstract}
- Purpose - Issues surrounding knowledge management, knowledge transfer and learning within organisations challenge continuity and resilience in the face of changing environments. While initiatives are principally applied within large organisations, there is scope to assess how the processes are handled within small and medium enterprises (SMEs) and to consider how they might be enhanced. This paper presents an evaluation of practice within an evolving software development unit to determine what has been learned and how the knowledge acquired has been utilised to further organisational development. These results provide the basis for the design and implementation of a proposed support tool to enhance professional practice.
\end{abstract}

- Design/methodology/approach - A small software development unit, which has successfully delivered bespoke systems since its establishment a number of years ago, was selected for analysis. The unit operates as a team whose actions and behaviours were identified and validated by the following means: in-depth interviews were carried out with each member of the team to elicit an understanding of individual and collective development. Interview data were recorded and transcribed and subjected to qualitative analysis to identify key themes underpinning knowledge acquisition and utilisation. Samples of project documentation were scrutinised to corroborate interview data. After analysing the data, a focus-group meeting was held to validate the results and to generate further insights into learning within the team.

- Findings - Qualitative analysis of the data revealed key changes in thinking and practice within the team as well as insight into the development of individual and collective contextual knowledge, tacit understanding and learning. This analysis informed the proposal of a bespoke, lightweight, web-based system to support knowledge capture and organisational learning (OL). This approach has the potential to promote resilience and to enhance practice in similar small or start-up enterprises.

- Research limitations/implications - Purposeful sampling was used in selecting a small software development team. This enabled in-depth interviewing of all members of the team. This offered a rich environment from which to derive awareness and understanding of individual and collective knowledge acquisition and learning. Focusing on a single small enterprise limits the extent to which the 
findings can be generalised. However, the research provides evidence of effective practice and learning and has identified themes for the development of a support tool. This approach can be extended to similar domains to advance research into learning and development.

- Practical implications - Results of the work undertaken so far have generated promising foundations for the proposed support tool. This offers software developers a system within which they can reflect upon, and record, key learning events affecting technical, managerial and professional practice.

- Originality/value - Small enterprises have limited resources to support OL. The qualitative research undertaken so far has yielded valuable insight into the successful development of a single software development team. The construction of a support tool to enhance knowledge acquisition and learning has the capacity to consolidate valuable, and potentially scarce, expertise. It also has the potential to facilitate further research to determine how the prototype might be extended or revised to improve its contribution to the team's development.

Keywords: Software development, Organisational learning (OL), Knowledge engineering, Knowledge management (KM), Small and medium enterprises (SMEs).

\section{Article Classification: Research paper}

\section{Introduction}

The research presented in this paper aims to evaluate the ways in which a small software development team has embraced emerging trends in the development of digital technologies in establishing and maintaining successful practice.

The team was selected because it represented an evolving environment promising a rich source of acquired knowledge and expertise. The aim of the research was to identify factors underpinning successful practice and to use the results of this analysis, if feasible, to develop a support tool to enhance future practice. The research identified evidence of tacit, contextual knowledge acquisition and utilisation and proposed a means of representing key facets of this learning in a web-based support tool designed to complement continuing tacit learning. It also has potential benefits in maintaining organisational resilience where individuals' skills may be lost; it can also serve to induct newcomers to the organisation.

Projects were identified as key sources of development, innovation and learning and formed the basis of the proposed support tool. Briefly, this is envisaged as a web-based repository of project templates capturing key insights into critical decisions and significant advances in current practice arising from work within individual projects. Team members will contribute summaries on the success and limitations of methods adopted in undertaking the project; they will also include links to related external locations where more detailed resources can be accessed, as presented in Section 7.

Section 2 outlines the relevance of Knowledge Management (KM), OL and SMEs to the research. Section 3 introduces the research methods used in the approach and Section 4 explains how the team evolved and identifies key factors that have supported individual 
and collective learning and development. Section 5 extends this analysis by discussing a focus-group meeting. Section 6 presents overall findings and Section 7 profiles a proposed support tool. Finally, Section 8 presents discussion and conclusions.

This paper offers insight into practice within a small, knowledge-intensive domain, which displays a range of learning characteristics that reflect effective OL capabilities such as flexibility, innovation and competitiveness. This understanding informs the proposal of a system that can enhance professional practice and knowledge transfer. Despite SMEs' economic contribution, they lack KM and LO resources and this paper serves the field by proposing a lightweight, web-based system to support KM and OL without placing onerous demands on practitioners' time. There is scope to extend this research to a wider range of SMEs to evaluate its potential benefits.

\section{Knowledge and Learning in Small and Medium-Sized Enterprises}

$\mathrm{KM}$ and OL concepts underpin this research, which is applied within an SME context, and themes emerging from the data analysis reflect developments in these fields.

$\mathrm{KM}$ is concerned with the nature and uses of knowledge within organisations (Nonaka and Takeuchi, 1995). Methods of capturing, reusing and exploiting knowledge assets impact upon the value and retention of organisational knowledge and expertise (Leonard-Barton et al., 2015). This research aims to identify the critical knowledge that has been acquired by the team and how has it been utilised. Addressing this question provides insight into how it might be retained and reused.

The nature and role of organisational knowledge is complex and contested (Newell and Swan, 2000) with different perspectives on its implicit embodiment within organisational infrastructure and individuals' actions (Lam, 2000; Teece and Al-Aali, 2011), or as a resource that is possessed (Alavi and Leidner, 1999). The question of how tacit knowledge can be represented explicitly and used to enhance practice is a critical element of this research.

IT-based systems are widely used to support KM initiatives (Fernandez and Sabherwal, 2015; Hislop et al., 2018) despite perceived limitations (Newell et al., 2006; Rus and Lindvall, 2002). Web-based technologies are proposed for knowledge capture and representation, as outlined in Section 7.

Vera et al., (2011) consider the relationship between individual and collective learning and identify the role of decision making and error detection and correction (Cohen and Sproull, 1996), as well as organisational evolution and transformation (Crossan et al., 1999), as significant OL developments. Analysis of the interview and focus-group data in Sections 4 and 5 provide evidence that these factors have influenced the team's development.

The distinction between tacit and explicit knowledge remains a critical factor within KM and OL. Tsoukas (2011) draws upon (Polyani and Prosch, 1975) in arguing that tacit knowledge cannot be articulated nor converted into explicit rules. It can be manipulated through dialogue to gain insight and understanding thereby generating new knowledge. The interplay between knowledge acquired through practical experience and interpersonal communication emerges as a significant theme from the research analysis. 
Unlike larger organisations, SMEs are less formal and bureaucratic yet more flexible and innovative (Turner et al., 2010) - characteristics evident in this research.

Software development is knowledge intensive and generates intellectual capital as a principal asset, and integrating KM into the software development cycle can improve the quality of system development (Panagiotou and Mentzas, 2011). There is evidence of its benefit in facilitating learning (Dingsoyr and Smite, 2014). A wide range of software engineering support tools are available, and repositories of shared documents, wikis, collaborative tools, and blogs facilitate $\mathrm{KM}$ in the software development process (Menolli et al., 2015; Shongwe, 2016). These activities implicitly entail individual and collective learning and the analysis of practice within the team demonstrates that collaboration, knowledge sharing and the collective use of development tools feature prominently.

\section{Research Methods}

This section outlines the data gathering and analysis methods underpinning the foundations of the support tool.

\subsection{Stages of the Research}

The research comprised the following stages: identifying the unit as the target domain; preparing a series of interview questions; conducting a series of recorded interviews with team members; transcribing the audio data; analysing the interview data to identify concepts relevant to the unit's learning; scrutinising a sample of project records to validate interview analysis; and holding a focus-group meeting to validate the themes derived from analysis of the interview data.

Generating reliable results from semi-structured qualitative interview data where beliefs elicited from a sample are presented as representative of a wider target population is contentious (Galvin, 2015). While larger samples are generally more reliable, samples of 5-6 participants are not uncommon in qualitative research. It is also important to be clear about the appropriateness of the sampling method used. This represents purposeful sampling which relies on the judgement of the researcher when it comes to selecting the units (e.g. people, pieces of data) to be studied (Patton, 2002; Palinkas et al., 2015). Purposeful sampling focuses on particular characteristics of a population that are of interest, which will best enable research questions to be answered. The aim of this research is to gain insights into the learning and knowledge acquired by all the team members (one manager and five software developers).

The interview questions were designed to elicit details about factors concerning individual and collective experience. They included observations on their notion of success e.g. what had been learned; what had led to improvements; and what had impeded progress.

The interviews were recorded and transcribed to generate a comprehensive data store; this was analysed to identify concepts relevant to the team's learning, development, and acquisition and utilisation of critical knowledge. A sample of historical project records was scrutinised to identify methods and procedures. Evidence from the sampling was cross-checked against interviewees' accounts of their experience. Project records were consistent with the evolving software engineering methods adopted by the developers, 
as addressed in Section 4. A focus-group meeting was held to validate the themes derived from the analysis of the interview data, presented in Section 5.

\subsection{Data Collection and Analysis}

The interview questions focused on general themes covering: development of the team; perceptions of the success and limitations of what had been achieved; details of approaches to software development e.g. methodologies adopted, issues concerning: risk, quality assurance, change management; and reflections on their individual learning and development. They also contained more probing follow-up questions. Individuals' perceptions of success and failure are qualitative observations in this context, acknowledging limitations in performance or outcome, and how the situation was dealt with, as well as recognising effective initiatives and their benefits.

\subsubsection{Interview and Project Record Data}

The interviews were recorded and transcript files were generated from the audio data. Qualitative research techniques were applied (Fink, 2003; Bryman, 2012; Silverman, 2013): key themes were extracted from interviewees' observations and analysed to generate an overall understanding of the team's structure and practice. This process of data reduction (Miles and Huberman, 1994) generated a detailed concept graph of significant factors in team development. Results are presented in Section 6.

A sample of archived project documentation was analysed to verify the observations derived from the interview data. Scrutiny of one of the earlier projects in the team's archive, to add a content management system to an existing website, revealed detailed design and quality standards documents consistent with quality assurance protocols governing system development. The effects of the change to a less rigid Agile approach were apparent in the records of a later project that built an on-line training website. Agile is a software application development method that advocates a more pragmatic and flexible approach to the delivery of solutions than the older waterfall method. Agile aims to deliver parts of the system, often without the next stage of development having been fully specified. Development continues as a series of iterations until an overall solution is achieved. Documentation from a recent project to merge two business systems into a single web application demonstrates how this approach is currently being implemented.

\subsubsection{Focus-Group Meeting Data}

The focus-group meeting aimed to validate the inferences drawn from the analysis and to elicit views about the findings and the implications for the team's development. Five broad themes were identified within which a series of specific questions were devised: The Team's Evolution; Project Knowledge; Individuals' Professional Development; Team Success Indicators, and A Proposal for a Knowledge and Learning Support System.

Analysis of a transcript file of the meeting validated findings and provided further evidence of evolving knowledge and capability, which is depicted in Figure 1.

\subsection{Summary}

The research study has been designed to answer three main questions: 
1. What critical knowledge and know-how have the practitioners acquired through the successful establishment and development of the team?

2. How have they learned to become effective practitioners?

3. Where might this knowledge reside and can it be elicited and represented to support $\mathrm{OL}$ and $\mathrm{KM}$ and thereby enhance professional practice?

The work presented in the paper addresses the first two questions and represents the basis upon which the third question can be answered by proposing a potential support system, as described in Section 7.

\section{Analysis and Results of the Interview Data}

This section presents analysis of the interview data, extended in Section 5 by incorporating insights derived from the focus-group meeting.

\subsection{Evolution of the Team}

The team was established by staff and students within a university computing department. Initially, students undertook software development projects for external clients. Team membership fluctuated but a core of former students established themselves into a more coherent entity. The portfolio of contracts has expanded and includes external clients as well as collaborative initiatives with internal partners.

Data analysis revealed key points where practice changed, and identified what was learned and what motivated the change at these stages e.g. perceived limitations with the approaches that were being used, or changes in behaviour resulting from previous mistakes and unsatisfactory project outcomes, or the challenges faced with issues such as code conflicts and program integration:

D1: "I would say initially when I started I didn't have the skills I need to actually do the job. But I don't have that concern now."

D1: "We started to explore solutions and one of the things that we came up with was source control. There are different solutions, originally we started with a source control solution called SPN. That didn't work, so we moved to Gitlab."

M: "In the early days we never used to say no, but now we do. If the project is risky or (there's) something we don't really understand, or we are not really getting along with the client, we just say no. Also I think my confidence has grown and my confidence in the team has grown too."

Arguably this has fostered a collective body of tacit knowledge which is now implicit in practice.

Changes in methodological approach, such as the adoption of peer programming in embracing Agile development methods (Dingsøyr, 2012; Nerur et al., 2005; Rigby et al, 2016), and improving the physical working environment by occupying an open-plan office, have encouraged collaboration, communication and the sharing of knowledge.

Analysis of the data revealed evidence of requests for advice, opinion and technical support from colleagues:

D2: "I think for the size of the team we are quite communicative with each other. Because we all sit together, there aren't many barriers to communication" 
M: "I think that as a team we realise each other's benefits and shortfalls. We are pretty good at sharing knowledge and ideas"

Their proximity to each other facilitated this interchange and reflects the interaction between factors such as layout, structure and culture that impacts on organisational development, (Argote, 2006). This is also consistent with 'transactive memory' (Wegner 1986, 1995) in which group members acquire knowledge about each other's capabilities, effective coordination and communication, and individuals' trustworthiness, which improves performance.

Evaluating the material suggests ways in which team members collectively shaped their own evolving environment e.g. moving away from an initially comprehensive documentation process during development to a more lightweight approach:

M: "The original document was big documentation. Myself and another member viewed it and we decided actually for the size of our organisation it was probably far too detailed, far too complex"

D1: 'We didn't want documentation, so for each project you would have several documents needing to be filled out before you even thought about coding'

This has been accompanied by embracing techniques such as Agile and Scrum (Schwaber, 1997; Noll et al, 2017). Scrum is a framework for organising teams within a software development environment, which is often adopted by organisations operating within an Agile context. This evolution has seen the team adapt techniques to meet their own particular requirements and style of working:

D2: "Scrum software development methodology basically tried to enforce every rule it possibly could from the methodology, which for the size of our team is a bit overbearing .... I think it is faster and easier to do (things) more informally."

However, engaging in Scrum did enable developers to improve some of their processes, for example they prioritised tasks more effectively, enhanced team working, and reduced the time taken to develop system components:

DS: "It's hard if you have a problem and you are in waterfall and you want to do a good job for the client, obviously it is more restricted than Agile. Agile is more flexible, as projects seem to flow better."

D1: "We took up Agile because it allows us to work quickly and get feedback from the client earlier on. It is more beneficial than after the entire project is finished, because it is much more expensive to change at that point."

As well as adapting methods and processes, other factors contributed to the team's success, such as learning from failure (Cohen and Sproull, 1996; Argyris and Schoen, 1978; Senge, 1994) and their ability to apply what they knew about computer software and development, to then build solutions for clients:

M: "What actually caused project failure was technical issues, it was not our fault; it was not the client's fault, but we couldn't do anything about the middle party (that was involved). That caused significant losses and at that point the client just lost confidence in our ability to do the project."

This was acquired by practice and engaging in projects continuously. Key indicators underpinning professional capability are identified as follows: 
- Individuals' professional development

- Technical and managerial evolution of the team - adoption of emerging methods and technologies

- Increasing professional experience and associated confidence and resilience

- Clients' satisfaction with solutions

- Establishment and consolidation of a stable and financially sound environment

Current practice reflects a flexible, Agile approach incorporating key software support tools. Significant knowledge resources reside in contextual practice as well as repositories such as a source code management tool (GitLab), project management software (ActiveCollab), and website analytics software (Sentry). GitLab is a system that stores projects' source code in repositories for code version control. It tracks changes and carries out continuous integration and delivery of products from within a single application interface. ActiveCollab is a web-based project management system which allows various project details to be recorded and shared between team members. Sentry is a real-time error tracking system recording errors and exceptions occurring on clients' websites. Dealing promptly with these issues helps build client trust:

D1: "As I work with clients day-to-day, being able to report issues and fix them before they're noticed has several benefits. One is that it builds a level of trust between us and our clients, knowing that we have everything under control."

This overview of key phases in the team's evolution appears in Figure 1 and shows that practice advanced along with changes in the wider software engineering community.

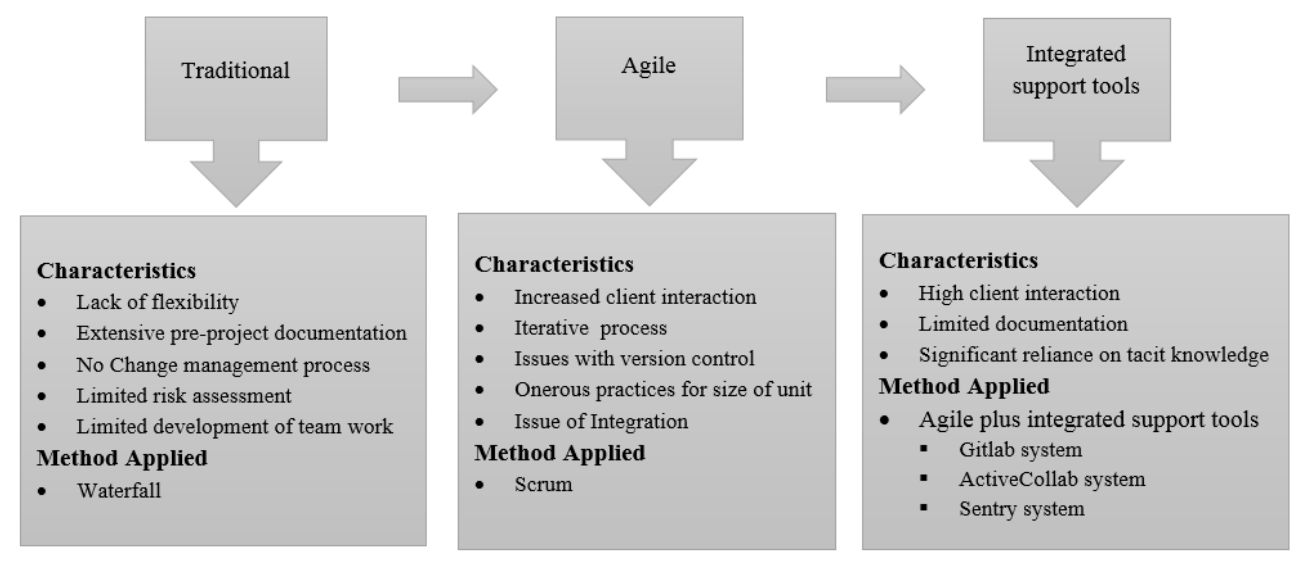

Fig. 1 Key phases of the team's development

This progression can be interpreted from the perspective of absorptive capacity, (Cohen and Levinthal 1989, 1990). Van Wijk et al. (2011) discuss the extent to which this construct, which entails the capacity to recognise the value of, assimilate and apply new external knowledge, complements the knowledge and learning that is central to organisational innovation and competitiveness.

\subsection{Individuals' Professional Development}

Individuals' technical skills have been complemented by soft skills including: communication, problem solving ability, cooperation and team work, which contribute to the overall effectiveness of the team. 
Data analysis pointed up team members' communication and collaboration in establishing tacit business protocols e.g. an ethos of open communication with clients, which helps mutual understanding of system requirements. This mitigates misunderstanding, reworks and client dissatisfaction. Collective decisions to adopt new approaches resulted from informed discussion and engagement with wider software practice. There is evidence of progress in learning how to handle clients' projects and to cost and schedule change requests:

D2: "We usually ask the client for feedback in the middle of the week and respond to requests at the end of the week. If we get too many requests from clients, I normally email them and inform that we will get back to them at the end of each week"

Team members take the initiative to learn specific tools and programing languages independently; they also experiment and check other external resources. A combination of observing external practice and individuals' studying, researching, experimenting and learning represents their primary problem-solving approach:

M: "Experimentation is something we all enjoy"

D3: "I am also keen on trying new technologies and having the right project in which to apply some of those new tools and techniques. In my free time I try to watch videos and learn new technologies and see where we can use them"

D1: "If we are trying to solve a problem there are two places where we look: the internet in general - one of our favourite websites is called stack overflow; or previous projects..."

Their increasing understanding of clients' business domains, allied to their insight into the benefits that technologies can facilitate, has enhanced the services provided to clients:

M: "With the HR system we built recently ... we got to challenge the client a lot. So after some time we had a good impact.... That got them questioning their own processes as well."

M: "Sometimes we have evolutionary projects. It does not necessarily have an end objective. People come to us without a definitive idea of what they want. ... Therefore, we have to work out how and what is the best way to get there."

Another significant professional skill is the reuse of project knowledge. The more they build and deliver projects, the more they have standardised formats which can be applied to new projects. Such techniques can have economic advantages. The introduction of Gitlab recently facilitated this skill.

They are able to learn from experience and to modify practice accordingly. For instance, when developing a system, they anticipate possible extensibility:

D2: "People want a system to suit their immediate needs but then a year or more later they come back and say, 'Can we extend this?' So you try to tease that out from the beginning so you don't end up writing something that ends almost road-blocking something you might want to do in the future."

From an OL perspective, these attributes demonstrate team members' acquisition of the core meta-abilities underpinning individuals' capacity to engage in OL, (Butcher et al., 1997; Selamat and Choudrie, 2007). These abilities comprise cognitive skills, selfknowledge, emotional resilience and self-drive. 


\subsection{A Rationale for Support}

The team represents a small environment whose members can communicate and work effectively. It was more fragmented and volatile in the early stages of its development with difficulties arising from limited experience and a higher turnover of staff. An attempt to capture valuable expertise and know-how would have benefits should staff leave and be replaced by newcomers. Capturing knowledge can also reinforce an understanding of limitations in practice and reasons for adopting new techniques.

The outcome of the initial data analysis was a proposal for a lightweight system to support knowledge capture and dissemination, and team learning. The system will focus on projects to encapsulate emerging knowledge and know-how. Details appear in Section 7.

\section{Data Validation and Further Insights}

Results of the analysis in Section 4 led to the proposal to develop the system outlined in Section 7. A focus-group meeting was held to validate the results and to gain further insights into current practice.

\subsection{Continued Team Learning}

The analysis presented in Section 4, was agreed to be consistent with team members' own views. A brief summary of perspectives on current activity is presented here.

Team members acknowledged the pressures they face in accomplishing the range of tasks for which they are responsible. This is likely to be symptomatic of other SMEs, particularly those working in knowledge intensive domains.

Apart from individuals' specific expertise, the value of a more general understanding of the business and regulatory contexts in which systems are developed were cited as critical to understanding client requirements and their associated business domains:

$\mathrm{M}$ : “...it certainly helps with clients because they feel we are on the same page with them because we understand their industry. That makes them trust us and make them tell us more, which means a better product is developed..."

Team members recognised that engagement with a range of projects had conferred insight into these facets of organisational structure and they were able to transfer knowledge across cognate domains. This helped facilitate their interaction with clients and to foster client trust.

The University partnership led to a request to integrate Virtual Reality (VR) development into their portfolio to complement academic interest in the area. This presents significant learning demands in terms of software development and user experience (UX) design and implementation.

Team members remained confident about their ability to meet this challenge, reflecting an awareness of the progress that had been made in adapting to new techniques and acquiring new skills:

D3: "I think doing various different projects: that improved our confidence through working with internal and external clients" 


\subsection{Reflections on Current Practice}

The focus-group meeting generated observations about perceived limitations with current practice plus opportunities for continued development and possible expansion. Briefly, these comprise extended use of GitLab resources, improved client communication and payment methods, and consultancy services to optimise clients' digital resources. These are indicative of the flexible, innovative qualities that are considered important for success (Van Wijk, 2011; Bettis et al., 2011; Wang and Ahmed, 2003).

Team members were aware of each other's strengths and weaknesses; they worked collaboratively and were mutually supportive. They had worked successfully across departments when undertaking internal institutional projects. This reinforces the view that they possess the core attributes to engage in OL (Butcher et al., 1997; Selamat and Choudrie, 2007).

One of the principal indicators of success was considered to be client satisfaction, represented by direct feedback and ongoing business relations:

M: "For us I think the most important success indicator is client's satisfaction ... If our customers are happy, ... then I think we're doing the right thing."

Some systems continue to evolve as additional functionality is requested in response to evolving business environments. An aspiration was to secure fewer, higher value contracts. This would be more cost effective as effort could be concentrated on significant pieces of work; currently, switching attention between a series of smaller projects can dissipate concentration and energy:

D3: "Project switching is an issue for developers... when you need to jump between projects it takes time for you learn where you're at, or where you've been. Working on two or three projects at a time causes complications and it takes us more time to do things. So if we had one bigger project, rather than many small ones, that would simplify the process."

This initiative poses questions about decision-making and risk, which are inherent in organisational development and change, and the proposed system can potentially support such strategic decision making.

\section{Overall Data Analysis Results}

Considering the three main research questions posed in Section 3.2, the answers derived from the work undertaken so far can be summarised as follows:

1. What critical knowledge and know-how have the practitioners acquired through the successful establishment and development of the team?

Sections 4 and 5 highlight the professional experience that individuals have gained and which underpins their success. Current work adopts modern software development methods and integrates automated tools to improve practice. This technical ability is complemented by an increased understanding of clients' business processes, which enhances team members' ability to optimise the application of digital technology to clients' business domains, as observed in Section 4.2. 
2. How have they learned to become effective practitioners?

The answer to question one is essentially that the team has learned and knows how to develop client software. This technical advancement is complemented by the absorption of tacit knowledge derived from practical experience and communication with colleagues to consolidate understanding, insight and know-how, as the analysis in Sections 4 and 5 suggests.

3. Where might this knowledge reside and can it be elicited and represented to support OL and KM and thereby enhance professional practice?

The answer to this question also follows from what has been found in answering the first two. Principally knowledge lies in the experience and practice of the individuals; it is manifested in software and more explicitly in program documentation e.g. plans, schedules, test reports etc. Key features of the team's progress lie in a complex relationship between factors which impact on successful practice e.g. insight, understanding, communication and motivation. Critical aspects of team knowledge can be articulated, particularly where this reflects an evaluation of processes and outcomes, for example.

So the aim, as outlined in Section 7, is to capture this kind of explicit knowledge through concise, considered observations on practice associated with individual projects and linked to additional resources. Video has been selected as the medium through which more general aspects of tacit knowledge and know-how can be conveyed.

\section{The Proposed System}

The proposed system focuses on projects to encapsulate emerging knowledge and knowhow. A key objective is to build a lightweight repository of core knowledge that does not impose onerous demands on team members updating the system.

The system will comprise a series of generic templates designed to reflect knowledge advancement and learning associated with specific projects. Templates will consist of sections for observations relating to principal project themes:

- Management

- Technical Matters

- Project Evaluation

Each of these will provide a concise summary of project characteristics, capturing key technical, managerial or other significant factors arising from the project. Currently information associated with projects resides in documentation within project support tools e.g. GitLab, ActiveCollab, and Sentry. Links will be provided to relevant documents, or components of the support tools, where more specific details, e.g. source code comments, can be accessed.

The aim is to capture critical learning associated with individual projects. This will reflect reasons for proposing change e.g. the introduction of version control methods, and indicate what prompts decisions as well as the outcomes when they are acted upon. There are potential benefits in such reflection (Falconer, 2006; Schoen, 1983).

This kind of knowledge can be complemented by more general know-how acquired through experience across projects. These are likely to include subtle behaviours such as dealing with clients, for example. This know-how can be conveyed effectively through a 
short video clip and it is anticipated that the system will be augmented by integrating relevant clips, accessible from a top-level dashboard, with additional links from project pages, as appropriate. Figure 2 presents a high level schematic representation of key system components.

Summary data recorded for some projects will be routine but details of unanticipated difficulties and how they were addressed will be more valuable. By encouraging team members to be critically reflective, recommendations can articulate viewpoints and explain key decisions e.g. recognition of version control issues mentioned in Section 4.1.

Similar recognition of the inherent knowledge encapsulated in project documentation, and its value as a learning resource, is addressed by (Holzmann et al., 2012). Ding and Liang et al. (2014) focus on the role and value of documentation in the software development process, asserting that the quality of documentation affects its usefulness, especially credibility, conciseness, and unambiguity, and that its retrieval and utilisation can improve the quality of software development.

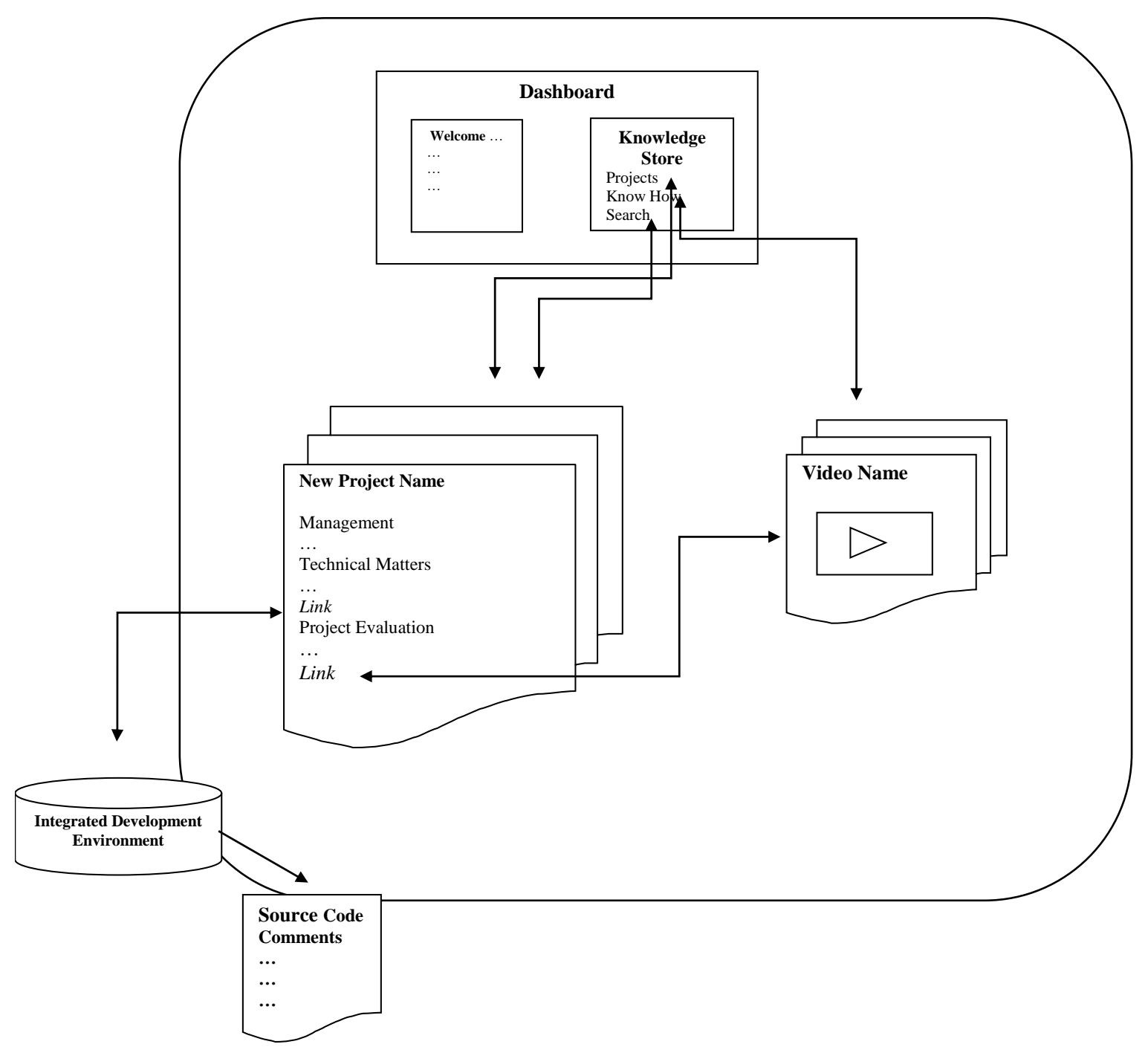

Fig. 2 Schematic Overview of System Prototype 
The user is presented with a top level dashboard containing information, navigational features and resources such as search, for example. The project repository is extended by developers and interrogated by team members and other interested users. Knowledge associated with individual projects comprises specific comments as well as associated detail located externally e.g. in relevant tools, documents, databases or blogs. This basic framework represents a starting point. Interface and functional features will be refined through an iterative development process in building an initial prototype. The prototype itself will form the basis for further development which is likely to result from system testing and user engagement.

\section{Discussion and Conclusions}

This research has been motivated by issues concerning knowledge management, knowledge transfer, and learning within organisations, that challenge continuity and resilience in the face of changing environments, particularly in small or start-up enterprises.

A detailed analysis of data gathered from a software development team has revealed the nature and extent of learning underpinning current practice. This informed the proposal of a bespoke system to support knowledge capture and OL in which project templates are to be used to record concise, reflective observations on key project features. This will record processes, methods and experiences that have been effective together with those that have been unsatisfactory or in need of improvement. Links to relevant resources will extend the associated knowledge base, as illustrated in Figure 2. Cumulatively, the project details will encapsulate details of the team's decision making and associated learning. The system will also enable potential newcomers to the team to orientate themselves with respect to the nature and scope of the work undertaken and the methods and approaches adopted in the software development process.

Information Technology continues to exert significant changes on society. The ubiquity of mobile devices and their increasing role in individuals' engagement in commercial transactions, communication and personal fitness, for example, are indicative of changing behaviour. Increasing automation in areas such as transport, for example, are likely to impact heavily on employment and the routine of people's lives. The application domain of this research is software development and the aim is to support software engineering practice. Contributing to the maintenance of good software development standards is essential to the establishment and continuity of a sound digital infrastructure.

Despite the constraints imposed by the scope of the project, the findings of the analysis of this particular small enterprise have provided valuable insight into organisational development in a knowledge-intensive environment. The implementation of a prototype software system will offer further research opportunities to discover how OL can enhance individual and collective practice. Advances in these areas are likely to be of interest to other members of the LO community engaged in learning initiatives, particularly within similar small and developing enterprises where valuable skills may be in short supply and the continuity of organisational knowledge is critical.

\section{References}

Alavi, M. and Leidner, D. E. (1999) 'Knowledge management systems: issues, challenges, and benefits', Communications of the AIS, 1(7), pp. 1-37. 
Argote, L. (2006) Organizational Memory. In Prusak, L. and Matson, M. (Eds) Knowledge Management and Organizational Learning: A Reader, Oxford: Oxford University Press

Argyris, C. and Schoen, D. (1978), Organizational Learning: A Theory of Action Perspective, Addison Wesley: Reading, MA.

Nunes, M.B., Annansingh, F., Eaglestone, B., and Wakefield, R. (2006) "Knowledge management issues in knowledge-intensive SMEs", Journal of Documentation, Vol. 62 Issue: 1, pp.101-119.

Becerra-Fernandez, I. and Sabherwal, R. (2015) Knowledge Management Systems and Processes, $2^{\text {nd }} \mathrm{Ed}$, Abingdon: Routledge

Bettis, R.A., Wong, S.S., and Blettner, D. (2011) Dominant Logic, Knowledge Creation, and Managerial Choice. In Easterby-Smith, M. and Lyles, M.A. (Eds) Handbook of Organizational Learning and Knowledge Management, Chichester: John Wiley \& Sons

Bryman, A. (2012) Social research methods Bryman, OXFORD University Press.

Butcher, D., Harvey, P. and Atkinson, S. (1997), Developing Business Through Developing Individuals, Cranfield University, Cranfield.

Chen, C. W., Chang, M., Tseng, C., Chen, B., and Chang, Y.Y. (2013) 'Critical human factor evaluation of knowledge sharing intention in Taiwanese enterprises', Human Factors and Ergonomics In Manufacturing, 23(2), pp. 95-106.

Choochote, K. (2013) 'Knowledge Management Strategyfor SMEs', International Journal of Advanced Computer Science and Applications(IJACSA), 4(8), pp. 167-172. Available at: http://ijacsa.thesai.org/.

Cohen, D, and Sproull, D, (1996) Organizational Learning. Sage, London,

Cohen, W.M., and Levinthal, D.A. (1989) Innovation and Learning: The two faces of R\&D. The Economic Journal, 99(397): 569 - 596.

Cohen, W.M., and Levinthal, D.A. (1990) Absorptive Capacity: A new perspective on learning and innovation. Administrative Science Quarterly, 35(1): 128 - 152.

Crossan, M., Lane, H., and White, R. (1999) An organizational learning framework: From intuition to institution. Academy of Management Review 24: 522-538.

Dingsøyr, T., Brede Moe, N., Nerur, S.P., and Balijepally, V. (2012) 'A decade of agile methodologies: Towards explaining agile software development' Journal of Systems and Software 85(6) pp. 1213-1221

Dingsoyr, T. and Smite, D. (2014) 'Managing knowledge in global software development projects', IT Professional, 16(1), pp. 26-29.

Durst, S. and Wilhelm, S. (2011) 'Knowledge management in practice: Insights into a mediumsized enterprise's exposure to knowledge loss', Prometheus (United Kingdom), 29(1), pp. 2338 .

Durst, S. and Edvardsson, I.R. (2012),"Knowledge management in SMEs: a literature review", Journal of Knowledge Management, Vol. 16 (6) pp. 879 - 903.

Falconer, L. (2006) "Organizational learning, tacit information, and e-learning: a review", The Learning Organization, Vol. 13 Issue: 2, pp.140-151.

Fink, A. (2003) How To Design Survey Studies,Sage Publication, London 21-22. 
Galvin, R. (2015) 'How many interviews are enough? Do qualitative interviews in building energy consumption research produce reliable knowledge?' Journal of Building Engineering, Volume 1, Pages 2-12

Han, T.-S., Chiang, H.-H. and Chang, A. (2010) 'Employee participation in decision making, psychological ownership and knowledge sharing: mediating role of organizational commitment in Taiwanese high-tech organizations', The International Journal of Human Resource Management, 21(12), pp. 2218-2233.

Hayes, N. (2011) Information Technology and the Possibilities for Knowledge Sharing. In Easterby-Smith, M. and Lyles, M.A. (Eds) Handbook of Organizational Learning and Knowledge Management, Chichester: John Wiley \& Sons

Hislop, D., Bosua, R. and Helms, R. (2018) Knowledge Management in Organizations $4^{\text {th }}$ Ed., Oxford, OUP

Holzmann, V., Mischari, S., Goldberg, S., and Ziv, A. (2012) "New tools for learning: a case of organizational problem analysis derived from debriefing records in a medical center", The Learning Organization, Vol. 19 Issue: 2, pp.148-162,

Jensen, J.A. and Rasmussen, O.E (2004) "An inquiry into the foundations of organizational learning and the learning organization", The Learning Organization, Vol. 11 Issue: 6, pp.478490.

Lam, A. (2000) 'Tacit Knowledge, Organizational Learning and Societal Institutions: An Integrated Framework', Organization Studies, 21(3), pp. 487-513.

Lee, M. R. and Lan, Y. C. (2011) 'Toward a unified knowledge management model for SMEs', Expert Systems with Applications, 38(1), pp. 729-735.

Leonard-Barton, D., Swap, W. and Barton, G. (2015) Critical knowledge transfer: tools for managing your company's deep smarts Boston: Harvard Business Review Press

Menolli, A., Cunha, M.A., Reinehr, S. and Malucelli, A. (2015) "Old" theories, "new" technologies: Understanding knowledge sharing and learning in Brazilian software development companies', Information and Software Technology, 58, pp. 289-303.

Menolli, A.L., Malucelli, A and Reinehr, S. (2011) Towards a Semantic Social Collaborative Environment for Organizational Learning, The 7th International Conference on Information Technology and Applications (ICITA 2011)

McQueen, R.J. and Janson, A. (2016) "Accelerating tacit knowledge building of client facing consultants: Can organizations better support these learning processes?", The Learning Organization, Vol. 23 Issue: 4, pp.202-217.

Miles, M.B. and Huberman, A.M. (1994) Qualitative Data Analysis, $2^{\text {nd }}$ Ed. Sage, Thousand Oaks, CA

Mullen, B. and Goethals, G R (Eds.), Theories of group behavior (pp. 185-205). New York: Springer-Verlag

Misra, A and S.C. (2014) Conceptual modeling for knowledge management to support agile software development, The Knowledge Engineering Review, Vol. 29:4, 496-511.

Nerur, S., Mahapatra, R., \& Mangalaraj, G. (2005). Challenges of migrating to Agile methodologies. Communications of the ACM, 48(5), 72-78.

Newell, S., Bresnen, M., Edelman, L., Scarbrough, H. and Swan, J. (2006) 'Sharing knowledge across projects: Limits to ICT-led project review practices', Management Learning, 37(2), pp. $167-185$. 
Newell, S. and Swan, J. (2000) 'Trust and inter-organizational networking', Human Relations, 53(10), pp. 1287-1328.

Noll, J., Razzak, M. A., Bass, J. M., \& Beecham, S. (2017). A Study of the Scrum Master's Role, Proceedings of Product-Focused Software Process Improvement (pp. 307-323). Springer, Cham.

Nonaka, I and Takeuchi, H. (1995) The Knowledge-Creating Company, OUP, Oxford.

Palinkas LA, Horwitz SM, Green CA, Wisdom JP, Duan N, Hoagwood K. Purposeful sampling for qualitative data collection and analysis in mixed method implementation research. Adm Policy Ment Health. 2015;42,533-544

Panagiotou, D. And Mentzas, G. (2011) 'Leveraging Software Reuse With Knowledge Management In Software Development', International Journal of Software Engineering and Knowledge Engineering, 21(5), pp. 693-723.

Patton, M. Q. (2002) Qualitative research and evaluation methods (3rd ed.). Thousand Oaks, CA: Sage.

Polyani, M. and Prosch, H. (1975) Meaning. Chicago: The University of Chicago Press

Prusak, L. (2001) 'Where did knowledge management come from?', IBM Systems Journal, 40(4), pp. 1002-1007.

Ravishankar, M. N. and Pan, S. L. (2008) 'The influence of organizational identification on organizational knowledge management (KM)', Omega, 36(2), pp. 221-234. 6.

Rigby, D.K., Sutherland, J. and Takeuchi, H. (2016) Embracing Agile, Harvard Business Review, May 2016 issue pp.(40 - 48, 50)

Rus, I. and Lindvall, M. (2002) 'Knowledge management in software engineering', IEEE Software, 19(3), pp. 26-38.

Schwaber K. (1997) SCRUM Development Process. In: Sutherland J., Casanave C., Miller J., Patel P., Hollowell G. (eds) Business Object Design and Implementation. Springer, London

Selamat, M.H. and Choudrie, J. (2007) "Using meta-abilities and tacit knowledge for developing learning based systems: A case study approach", The Learning Organization, Vol. 14 Issue: 4, pp.321-344.

Senge, P. (1994), The Fifth Discipline: The Art and Practice of a Learning Organization, Nicholas Brealey Publishing, London.

Schön, D.A. (1983), "From technical rationality to reflection-in-action", The Reflective Practitioner How Professionals Think in Action, Basic Books, New York, NY, pp. 21-75.

Shongwe, S. (2016) Knowledge Management in Software Development: A Literature Review International Conference on Intellectual Capital and Knowledge Management and Organisational Learning; Kidmore End: 311-317. Kidmore End: Academic Conferences

Silverman, D. (2013) Doing Qualitative Research: A Practical Handbook, SAGE Publications.

Teece, D.J. and Al-Aali, A. (2011) Knowledge Assets, Capabilities and the Theory of the Firm. In Easterby-Smith, M. and Lyles, M.A. (Eds) Handbook of Organizational Learning and Knowledge Management, Chichester: John Wiley \& Sons

Tsoukas, H. (2011) How should we understand tacit knowledge? A Phenomenological View. In Easterby-Smith, M. and Lyles, M.A. (Eds) Handbook of Organizational Learning and Knowledge Management, Chichester: John Wiley \& Sons 
Turner, R., Ledwith, A. and Kelly, J. (2010) 'Project management in small to medium-sized enterprises: Matching processes to the nature of the firm', International Journal of Project Management, 28(8), pp. 744-755.

Van Wijk, R., Van Den Bosch, F.A.J., and Volberder, H.W. (2011) Absorptive Capacity Taking Stock of its Progress and Prospects. In Easterby-Smith, M. and Lyles, M.A. (Eds) Handbook of Organizational Learning and Knowledge Management, Chichester: John Wiley \& Sons

Vera, D., Crossan, M. and Apaydin, M. (2011) A Framework for Integrating Organizational Learning, Knowledge, Capabilities, and Absorptive Capacity. In Easterby-Smith, M. and Lyles, M.A. (Eds) Handbook of Organizational Learning and Knowledge Management, Chichester: John Wiley \& Sons

Wang, C.L. and Ahmed, P.K. (2003) "Organisational learning: a critical review", The Learning Organization, Vol. 10 Issue: 1, pp.8-17.

Wegner, D.M. (1995). A Computer Network Model of Human Transactive Memory. Social Cognition: Vol. 13, No. 3, pp. 319-339.

Wegner, D. M. (1986). Transactive memory: A contemporary analysis of the group mind. In

Wei Ding, A., Peng Liang, A., Tang, A. and van Vliet, H. Knowledge-based approaches in software documentation: A systematic literature review, Information and Software Technology 56 (2014) 545-567. 